\title{
La ciencia en el desarrollo de América latina
}

\begin{abstract}
AMILGAR O. HERRERA fue profesor de la Facultad de Ciencias Exactas y Naturales de la Universidad de Buenos Aires, y Vicepresidente del Instituto Nacional de Geologia y Mineria de la República Argentina hasta 1966. Desde agosto te ese año es Profesor Investigador de la Facultad de Ciencias Fisicas y Matemáticas de la Universidad de Chile. En este artículo expone algunas de las ideas que desarrollarí en su libro a publicarse por el Instituto de Estudios Internacionales, sobre una Política Cientifica para América latina.
\end{abstract}

“...Los problemas materiales del mundo pueden ser resueltos por la ciencia, y los factores que inhiben un desarrollo racional son políticos, sociales y psicológicos, y no técnicos en el sentido material. Se acostumbraba a decir que la política es el arte de intentar sollo lo que es posible; ahora, desde que casi todlas las cosas son físicamente posibles, la política es cada vez mís el arte de encontrar explicaciones de por qué no se hacen"(The Science of Science, Londres, 1966, p. 10).

El atraso relativo de América latina respecto de los países desarrollados, en todo lo que se refiere a investigación científica y tecnologica, es demasiado conocido para que sea necesario describirlo en detalle. Es necesario destacar, sin embargo, que la diferencia en cantidad y, calidad de la producción científica no es más que un aspecto de la brecha que separa a nuestros países de los más desarrollados en este terreno. En los países adelantados la mayor parte de la investigación científica y tecnológica se realiza en relación con temas que directa o indirectamente están conectados con sus problemas de desarrollo. El progreso científico se refleja en forma inmediata y espontánea en el funcionamiento de sus fábricas, en su tecnología agrícola, en su infraestructura $y$, en general, en el constante incremento de la producción.

En América latina, por el contrario, la mayor parte de la investigación científica que se efectúa guarda muy poca relación con las necesidades más apremiantes de la región. Para demostrarlo basta hacer una breve reseña de la situación en un campo que es todavía vital para la economía de prácticamente todos los países del área; el de los recursos naturales. En el caso de los recursos minerales es bien sabido que el mapa geológico general de un país constituye la base indispensable para cualquier plan regional de evaluación y prospección minera. Con algunas limitaciones, se puede decir que el cono- 
cimiento que tiene un país de su potencialidad en recursos minera. les es proporcional al conocimiento que posee sobre sus condiciones geológicas. En América.latina se puede estimar que menos del 5 por ciento del área total ha sido mapeado geológicamente en las escalas apropiadas ${ }^{1}$. Como contraste conviene recordar que Canadá, con una superficie de casi $10.000 .000 \mathrm{Km}^{2}$, ha mapeado el 75 por ciento de su territorio en esas escalas. En lo que se refiere al inventario y evaluación, ninguno de los países de la región posee un inventario físico adecuado de sus recursos minerales conocidos, es decir, un inventario con información actualizada y completa sobre el tipo, calidad y.características geológicas de las reservas de cada uno de los productos mineralés de interés económico. Estas deficiencias de información se refieren a los recursos minerales más importantes, generalmente metales y combustibles. El conocimiento sobre los restantes es menor aún. Se puede afirmar que sobre más del $70 \%$ de los productos minerales que el Mincral Facts and Problems del U.s. Bureau of Mines, registra como importantes para la industria moderna, la información existente es nula o demasiado general para ser de utilidad práctica ${ }^{2}$.

En otro recurso natural que es esencial para el desarrollo dé América latina, el suelo, la situación es similar a la que hemos descrito para los recursos minerales. Solamente entre el 8 y el 10 por ciento de los suelos de la región han sido mapeados en escalas de reconocimiento. Los mapas semidetallados abarcan entre el 4 y el 5 por ciento del área, y los detallados el 1 por ciento ${ }^{3}$. Además, la falta generalizada de investigaciones edafológicas básicas y coordinadas hace que, en muchos casos, no pueda saberse el significado de las unicladés que aparecen en el mapa, por lo.que éste "puede no servir así más que de insólita decoración mural" ". La gravedad de estas deficiencias en el conocimiento de los suelos se refleja claramente en la siguiente afirmación del informe ya citado: "Es necesario tener en cuenta, en estos momentos en que se pretende programar el desarrollo latinoamericano, que no es posible pensar en una planificación que en la práctica se traduzca en mejoramientos concretos de la productividad agropecuaria, si no se cuenta con el apoyo de una információn completa y segura sobre los suelos. Hasta ahora los países de la región no disponen de esa información y además carecen de medios adecuados para obtenerla a breve plazo".

\footnotetext{
: ${ }^{H}$ Herrera, A. O. Los Recursos Minerales de Amórica latina. Editorial Universitaria de Bucnos Aires, 1965 , p. 43.

2Op. Cil., p.'39.

sLos Recursos Naturales en América latina, su conocimiento aclual $c$ investigaciones necosarias en ese campo. v - Los suelos. CEPAL r/cN. 12/670/Add 5, 1953.

LOp. Cit.
} 
La situación descrita, y que se repite en el caso de los recursos forestales, el agua subterránea, etc., se refiere sólo a la fase más primaria de la investigación de los recursos naturales, que es la de realización del inventario físico. En la etapa industrial propiamente dicha, es decir, en las fases de elaboración y transformación, se observan las mismas deficiencias. La investigación tecnológica es muy escasa y "con gran frecuencia se limita a considerar las posibilidades de utilización industrial de los recursos naturales de la región en el ámbito clel laboratorio, sin pasar a la etapa de planta piloto y sin considerar, por lo tanto, los aspectos técnicos y económicos de la transformación inclustrial'"5.

En lo que se refiere al desarrollo industrial en general, las condiciones no son mejores. No existe prácticamente investigación tecnológica al nivel de las empresas. La mayor parte de la industria latinoamericana se estableció en base a la transferencia de técnicas provenientes de los países más clesarrollados, sin que se realizara el mínimo de investigación tecnológica necesario para adaptarla a las condiciones locales. Los institutos tecnológicos cle la región, financiados en su mayor parte por los Estados, no amplian, en general, sus actividades al asesoramiento de la inclustria existente para la solución de los problemas de rutina ${ }^{6}$.

La investigación básica que se realiza en la región tiene lugar principalmente en las universiclades o en institutos relacionados con ellas o con organismos èstatales. Salvo raras excepciones, sus planes de investigación no guardan ninguna relación con las necesiclacles de la industria, o con los problemas generales del desarrollo económico de la región. Por otra parte, aun en los casos en que pudiera existir esa relación, faltan en América latina facilidades para llevar la investigación a una etapa en que pueda servir de apoyo efectivo a la industria ${ }^{7}$.

La característica más importante del cuadro que acabamos de esbozar tan brevemente, es la escasa relación existente entre la actividad científica y tecnológica del área y los problemas básicos cle desarrollo que ésta enfrenta. Insisto sobre este punto porque esta situación, tanto o' más que el volumen absoluto de investigación que se realiza es, en mi opinión, un carácter distintivo del subdesarrollo en el campo que estamos considerando. Volveré más sobre este tema al tratar lạs causas del atraso científico y tecnológico.

\footnotetext{
Informe final de la conferencia sobre la aplicación de la ciencia y la tecnologia al desarrollo de América latina, p. 41, unesco, Santiago de Chile, 1965.

OOp. Cit., p. 41.

TOp. Cit., p. 41.
} 
¿Cuáles son las consecuencias de estas diferencias en la cantidad, calidad y dirección de la investigación cientifica en América latina, con respecto a la que se realizan en los países desarrollados? No existe una manera dirẹcta de medir los efectos del avance científico y tecnológico en el progreso total de una sociedad, pero se pueden usar medios indirectos para apreciar su influencia en el crecimiento económico. Los más útiles son los índices de productividad y de crecimiento de la productividad, especialmente la producción por persona ocupada ${ }^{8}$. Según datos de la CEPAL, en los últimos diez años, mientras el producto por persona activa crecía en América latina escasamiente al '2 por ciento por año, en los Estados Unidos lo hacía a un ritmo sensiblemente mayor, y en Europa Occidental y Japón esa tasa se elevaba al 4 y 6 por ciento por año. Un fenómeno similar se producía en los países socialistas. En lo que se refiere a la actividad agropecuaria, en Estados Unidos y Europa Occidental el producto por persona ocupada ha estado creciendo a un ritmo de 5 y 6 por ciento, mientras que en América latina, y para un periodo particularmente favorable, sólo ha crecido a una tasa anual de poco más del 2 por ciento. Estas tasas de crecimiento de la productividad son suficientemente alarmantes por sí solas, pero lo son mucho más si se considera que América latina parte de índices de producto medio por persona ócupada que representan entre un tercio y un séptimo del que tienen los paises adelantados ${ }^{10}$.

¿Cuáles son las causas del rețraso científico y tecnológico de América latina? Es obvio que la respuesta correcta a esta pregunta es de importancia funclamental, ya que es cl prerrequisito indispensable para el planeamiento de cualquier política que tienda a superar ese attraso.

Las respuestas que se clan más comúnmente a esta interrogante, son de clos tipos. El primero radica las causas clel retraso en alguna condición básica, inherente a los pueblos de América latina, que los incapacita para el progreso material. Se expresa diciendo que los latinoamericanos o los latinos en general, según la procedencia del opinante, no tienen la predisposición para la técnica que es tan característica de los países anglosajones. El segundo tipo, muy popular en

\footnotetext{
8Atomation and Economic Progress. Edited by H. R. Bowen and G. L. Mangun, Prentice Hall, Inc., 1966 .

"Discursos de M. Balboa en la Conferencia sobre la aplicación de la ciencia y la tecnologia al Desarrollo de América latina, unzsco-CEPAL, 1965.

${ }^{10} \mathrm{Op}$. Cit., p. 2.
} 
reuniones de cientificos, conferencias internacionales, etc., acude a razones mucho más circunstanciales: falta de fondos por incomprensión e ignorancia de los gobiernos, trabas burocráticas, incomprensión general de la sociedad latinoamericana de la importancia de la ciencia, etc.

Para refutar el primer tipo de argumentos, basta recordar que lo mismo se dijo de los eslavos, prícticamente hasta que la Unión Soviética lanzó su primer Sputnik derribanclo el mito; de los asiáticos en general hasta que Japón, en 1905, derrotó militarmente a una de las mayores potencias occiclentales, y cle los chinos, en particulaŕ, hasta que cletonaron su primera bomba cle hidrógeno en un tiempo consiclerado record aun para una potencia científica de primer orden. Pese a la evidente falacia del argumento, sin embargo, se scguirai sosteniendo para América latina, inclusive por personas de la región, laasta que los latinoamericanos prueben, con hechos inclubitalbles, que se trata de uno cle los tantos mitos que se han esgrimiclo a través de la historia para ocultar las verdacleras causas de la miseria y la opresión cle grancles sectores de la humaniclad.

El segundo tipo de explicáción está mucho mils cerca de la realiclad, pero no va al fonclo del problemi. En efecto, es cierto que en América latina los gobiernos y los sectores dirigentes no apoyan sulicientemente el clesarrollo científico y que esto se traduce en escase\% de fondos, trabas burocráticas, falta de comprensión del papel de la ciencia en la sociedacl, etc. Pero esta explicación toca solamente los efectos más visibles cle causas que están prolunclamente enraizaclas on las condiciones socioeconómicas de la sociedad latinoamericana. Su defecto principal es que trata el problema clel atraso científico y tecnológico en forma aislacla, sin relacionarlo estrechamente con los factores esenciales que condicionan el subdesarrollo general de la región.

El impulso a la investigación científica y tecnológica sc produce principalmente por dos caminos: el Estado, en la medida en que éstc trata de alcanzar los grandes objetivos que se plantea la sociedad, y el cmpresariado industrial, que en su cleseo de aumentar continuamente su nivel cle productividad y de eficiencia, genera e impulsa la investigación tecnológica que transfiere en beneficio de la sociedad los resultados de la investigación científica.

Como es bien sabido, la industrialización de América latina fue principalmente el producto de contingencias originadas fuera de la economía latinoamericana y de sus centros de decisión. Según Osvaldo Sunkel11. "El fenómeno de la industrialización comienza a acelerarse

"1Sunkel, Osvaldo. El marco historico del proceso de desarrollo y subdesarrollo. Cuadernos de ILPES, Serie Ir, Anticipos de investigación, No 1, Santiago de Chile, 1967. 
Amilcar O. Herrera / La ciencia en el desarrollo de América latina

en América latina desde la Primera Gierra Mundial y recibe un nuevo impulso a partir de la crisis de 1930, que se renueva a raíz del segundo conflicto bélico. Con posterioridad a la Segunda Guerra Mundial, ya se transforma én una política cleliberada en prácticamente todos los países de América latina". La industrialización se basó, casi exclusivamente, en la sustitución de importaciones, con la importación directa de tecnologías provenientes cle los países desarrollados, o con la aplicación cle soluciones pragmáticas con poca o ninguna investigación técnica.

Como consecuencia de este mecanismo de inclustrialización originaclo en acontecimientos externos a la región, el acceso gradual de la clase media al pocler político que se registra en Ámérica latina en las últimas clécaclas no fue acompañado, como sucelió en Europa durante la Revolución Industrial, por el pase a una sociedad con cambios institucionales aclaptaclos al crecimiento industrial: "...dos o tres clécadas de este tipo de crecimiento industrial "accidental" no ham resultaclo en la creación cle una cultura industrial que sea posible consiclerar como una alternativa al complejo cultural tradicional de laș clatses superiores. En América latina la industrialización no es ni cl producto de la actividad de una burguesía industrial ascendente, ni la ha produciclo"12. La clase empresarial surgida de este proceso ha siclo descrita en sus masgos esenciales por Marcos Kaplan"13: "Se trata de un empresariatclo que aparece y se clesarrolla tardíamente; en número limitado por la cstratificación social rígida; frenado por, a la sombra de, o en ensamblamiento con fuerzas tradicionales y monopolistas del país y clel cxtranjero; con escasas posibilidades de competitividad y capitalización. Este sector tiende a preferir las actividades mercantiles $y$ especulativas a las que requieren grandes inversiones tecnológicas. Suele progresar como empresariado político o de coyuntura, a impulso de alternativas institucionales y conmocionales sociales y bajo protección cle determinados grupos en el poder. Carece frecuentemente de clisciplina y ascetismo; prefiere la acumulación veloz y el consumo a la inversión productiva, sin justificar sus beneficios por la capitalización racionalizada $y^{\prime}$ por la difusión de beneficios sociales y nacionales. Su horizonte no excede los ámbitos de lo mercantil y dinerario... No representa ni transmite lo que merezca preservarse del orden tradicional, ni opera como vehículo de innovación".

${ }^{12}$ Véliz, Claudio, Obslacles to change in Latin Amcrica, Edited by C. Véliz Oxford University Press, D. 6, 1965.

t3kaplan, M. Paises en desarrollo y empresas públicas, Ediciones Macchi, Buenos Aires, 1965 , p. 35. 
Para considerar muy brevemente la acción del Estado como impulsor del desarrollo científico y tecnológico, conviene dividirla en dos periodos: el que va desde la independencia hasta aproximadamente la Primera Guerra Mundial, y el que le sigue hasta la actualidad. En el primer periodo se produce lo que se ha denominado "crecimiento hacia afuera". El crecimiento económico dependió esencialmente de la actividad productora de materias primas para la exportación, que se desarrolló sobre la base de là inversión extranjera en la mayoría de los países de la región. Si bien en este período los sistemas productivos se expandieron considerablemente en respuesta a la demanda exterior, esto se hizo más mediante una explotación más extensiva de los recursos naturales y de la mano de obra, que por la introducción de innovaciones tecnológicas. El desarrollo clel sector manufacturero fue muy escaso, porque los recursos obtenidos del sector básico de exportación permitían obtener bienes manufacturaclos de los países adelantaclos a precios relativamente convenientes. En términos generales, los recursos provenientes dcl sector exportador no se canalizaron para desarrollar otros sectores más clinámicos cle la economía, en gran parte porque "el Estado se convirtió abiertamente en el sirviente de las clases dominantes, los propietarios nacionales y extranjeros en el sector exportador, sus sostenedores urbanos, y los propietarios de la tierra, quienes estaban frecuentemente conectados con el comercio de exportación"14.

En el período que sigue a la Primera Guerma Mundial, se acelera la industrialización de América latina en las concliciones que ya hemos visto: sustitución de importaciones, trasplante de tecnologias importadas, etc. El Estado tuvo un papel importante en cste proccso, pero no como resultado de decisiones cleliberaclas de parte de una élite burocrática o política, sino como respuesta a situaciones impuestas por circunstancias externas ${ }^{15}$. Las causas de esta actitud son claras. Las clases altas, en general propietarias de la tierra y conectadas con el sector exportador, no tenían interés en apoyar los procesos de modernización de la cconomía. La clase media, cuyo ascenso coincide aproximadamente con el periodo de industrialización, llega al poder con "partidos reformistas que con muy pocas excepciones, fueron predominantemente urbanos, partidarios del comercio libre, liberales, radicalmente anticlericales y no industriales"10. El resultado, sobre la

IPinto, Anibal. Obstaclss to change in Latin Anerica, Edited by Chudio Véliz, Oxford University Press, 1967, p. 19.

${ }^{20} \mathrm{Op}$. Cit., p. 12: p. 3 .

1oVéliz, Claudio, Obstacles to change in Latin Amcrica, Oxford University Press, 1967, 
actividad del Estado, de esta contradicción entre la filosofía política que lo informa y las necesidades impuestas por los nuevos factores que afectan la economía mundial y la de la región, han sido descritas por Kaplan1ึ: "El Estado de los países latinoamericanos ha heredado y continúa una tradición secular de "leseferismo" liberal, que presenta su intervencionismo como anormal y transitorio, e incapacita al gobierno para plantear y resolver los problemas de acumulación e inversión eficiente clel ahorro nacional, de la extensión del mercado interno y de la regulación de las transacciones externas. 'Las estructuras gubernamentales tienden, cacla vez más, a carecer de estabiliclad, eficacia y prestigio, hasta para cumplir las funciones y servicios tradicionales. El proceso de cambio, la multiplicación de tareas nuevas, la insuficiente capaciclad política y adiministrativa del Estado para cumplir responsabiliclades ampliadas, paralizan o desvirtúan sus decisiones y sus actos, agralyan su inestabilidad, su ineficiencia y su clesprestigio".

La calracterización del Estado y del empresariaclo latinoamericano que acabamos cle ver es, sin clucla, algo esquemático, ya que hay excepciones, tanto en la acción del Estado como en la del empresariado industrial; pero es suficientemente exacta, descle el punto de vista de la situación general preclominante en la región, y basta para explicar su atuaso científico y tecnológico. En efecto, la incapacidad del Estado para canalizar la energía creaclora de los pueblos en función de objetivos nacionales propios, redujo su acción en el campo cultural, en el mejor de los casos, al apoyo de la educación y al fomento de las profesiones necesarias para el funcionamiento de una sociedad esencialmente estática - medicina, derecho, ingeniería en el sentido profesional, etc.-, clescuiclando casi totalmente la actividad científica más creadora. Esta ineficacia del Estado, unida a la falta cle una industria tecnológicamente progresista, explica tanto el escaso volumen de la investigación científica, como su desconexión con los problemas de la región. Como señalan Urquidi y Lajous ${ }^{\mathrm{ts}}$ para México, aunque la afirmación es válida para tocla América latina: "Puede decirse que en general, los hombres de ciencia, al reaccionar ante esta situación, radicalizaron su posición, es decir, prefirieron dedicarse a llevar a cabo investigación básica o pura y no a efectuar aquella que tuvicra que ver con la industria o con el gobierno o en general con la vida económica del país".

\footnotetext{
17Kaplan, Marcos, Desarrollo sociocconómico y estructuras estalales en América lalina, Aportes, Instituto Latinoamericano de Relaciones Internacionales, Paris, 196i, p. 31.

18V. Urquidi y Lajous, Educación superior. Ciencias y Tecnología en el Desarrollo Económico de México.
} 
Se plantea entonces el interrogante de cómo sacar a América latina del atraso científico y tecnológico que incide tan decisivamente sobre sus posibilidades de desarrollo. Sin embargo, si bien existe acuerdo general entre los científicos sobre la imperiosa necesidad de llevar la investigación científica y tecnológica de la región a niveles compatibles con los de una sociedad moderna, no existe la misma unanimidad entre los economistas y políticos. No se trata, por supuesto, de que éstos últimos se opongan a la incorporación de los beneficios de la ciencia moderna, sino de que muchos de ellos creen que ese objetivo se puede lograr por otros medios que no sean la creación de una elevada capacidad científica $y$ tecnológica propia. Esta posición se basa en que consideran que el desarrollo científico, al nivel cle los países adelantados, es demasiado caro, difícil y a largo plazo, por lo que está fuera de nuestras posibilidades en el futuro previsible. Si bien las alternativas que propone esta posición son bastante variadas, se pueden resumir en las dos proposiciones siguientes: a) Existe en el mundo desarrollado un enorme volumen de conocimiento técnico disponible para los países subdesarrollados. Este conocimiento puede adquirirse mediante patentes, o a través de la radicación de instalaciones industriales modernas provenientes del extranjero. Estos centros modernos de producción impulsarán el progreso tecnológico de la región mediante su influencia sobre el medio ambiente, es decir, por el conocido "efecto de demostración"; b) la investigación tecnológica en los países desarrollados está dirigida a la creación cle tecnologias que hacen uso intensivo del capital. En América latina se necesitan "tecnologías intermedias", con mayor uso de mano de obra, debido al problema del desempleo crónico. En consecuencia, no conviene introducir las tecnologías más avanzadas, y por lo tanto sólo necesitamos una ciencia y una tecnología también "intermedias", más baratas y acordes con nuestras posibilidades.

Teniendo en cuenta que las alternativas enunciadas a la creación de una ciencia y una tecnologia propias de alto nivel siguen discutiéndose en los círculos políticos y económicos más importantes de la región, es necesario analizarlas brevemente antes de plantearnos el problema de la formulación de una política de desarrollo cientifico. La primera posibilidad, es decir la renuncia a la creación cientifica en el nivel que se realiza en una sociedad moderna, para tratar de alcanzar el progreso material mediante la copia o la compra de sus resultados, nos obliga a reconsiderar el concepto mismo de desarrollo. En efecto, esa posición implica suponer que el progreso de los 
países subdesarrollados se puede obtener simplemente mediante el aumento de la producción, especialmente en ciertos sectores básicos de la economía, como siderurgia, petroquímica, etc., sobre el supuesto de que lo demás viene prácticamente solo. Supone, en el fondo; un concepto mecanicista primario del desarrollo. Creo, en cambio, que el desarrollo implica un proceso cleliberado mucho más profunäo y más amplio. Sunkel19 lo define así: "... el concepto de desarrollo, cuando éste se concibe como proceso de cambio social, se refiere a un proceso deliberado que persigue como finalidad última la igualación de las oportunidades sociales, políticas y económicas, tanto en el plano nacional como en relación con sociedades con patrones más elevados de bienestar material... La posición adoptada implica, en consecuencia, la necesidad de examinar y de buscar en la propia realidad latinoamericana y en la influencia que ésta sufre por el hecho de coexistir, con sociedades desarrolladas, el proyecto de nación y las formas de organización que habrán de satisfacer las aspiraciones de los grupos en nombre de los cuales se realiza la tarea de desarrollo". Helio Jaguaribe"20, refiriéndose a los estudios sobre desarrollo económico llevados a cabo desde 1930 dice: "se comenzó a comprender que el clesarrollo es un proceso social total cuyos aspectos culturales y politịcos no son menos relevantes que los económicos". Concebido así el proceso de clesarrollo, como una transformación profunda, y como una af̣irmación de la personalidad nacional, es evidente que sólo puede efectuarse si se ponen en juego todas las energias, toda la capacidad intelectual de un pueblo. Renunciar entonces a la creación científica, una de las manifestaciones básicas de la voluntad creadora cle unạ sociedad, para convertirse en meros apéndices intelectuales de los países adelantados, es renunciar a la posibilidad misma del desarrollo.

Por otra parte, la suposición de que el mero trasplante de tecnologías provenientes de países clesarrollados puede resolver los problemas. materiales del subdesarrollo, aun a costa de la subordinación intelectual, es errónea, y para probarlo basta examinar la experiencia ya existente. Como señala acertadamente Víctor Urquidi21: "América latina ha estado importando tecnología por más de 450 años y $\sin$ embärgo, aún ahora los oasis de modernismo tecnológico se destacan en un

${ }^{19}$ Sunkel, Osvaldo, El concepto de desarrollo, Capitulo 11. Preliminar, ILpes, 1966.

suaguaribe, H., Obstacles to change in Latin America, Edited by Claudio Véliz, Oxford University Press, 1967, p. 184.

"Urquidi Victor, Obstacles to change in Latin Anterica, Edited by Claudio Véliz, Oxford University Press, 1967, p. 102. 
vasto desierto de atraso e ignorancia". Manuel Balboa ${ }^{22}$, refiriéndose al mismo problema, dice: "se presenta en América latina la aparente contradicción de que la aplicación de tecnologias modernas es incompatible con los objetivos de elevación de los niveles de empleo productivo y un cuadro en que los conocimientos aparecen penetrando en determinados sectores o en determinadas áreas, cuya lenta difusion revela la imagen de verdaderos enclaves sectoriales y espaciales, vinculados frecuentemente con las actividades de exportación y de sustitución de importaciones. No inciden ellos sensiblemente, en el tiempo que fuera dable esperar, en el mejoramiento general del producto y del ingreso...". En lo que se refiera a la incapacidad de los enclaves de tecnología moderna para impulsar por sí solos el conocimiento tecnológico, se pueden citar en América latina muchos ejemplo, pero unos pocos son suficientes. La economía de Bolivia ha estado condicionada a la producción cle estaño descle principios de este siglo, y sus minas exan explotadas por compañías que utilizaban métodos modernos de exploración y explotación. Cuando se nacionalizaron en 1952, la mayoría de los técnicos extranjeros que dirigían las operaciones salió del país. Los resultados para la producción y para el desarrollo de nuevas reservas fueron desastrosos, en gran parte porque Bolivia no contaba con el personal técnico necesario para una operación eficiente, a pesar del medio siglo de explotación intensiva de sus recursos minerales por empresas extranjeras. El caso de los productos tropicales es también significativo. Varios de los países de la región son proveedores de productos tropicales en el mercado internacional, y la explotación la realizan en parte grandes empresas extranjeras que cuentan con todos los recursos de la tecnología nioderna. En esos países, sin embargo, se conoce muy poco sobre las características básicas de los suelos tropicales, lo que constituye un obstáculo casi insalvable para cualquier intento de diversificación de la producción en función de cambios en la demanda externa o interna. En la industria manufacturera, el resultado del trasplante de tecnologías ha sido descrito, entre otros, por Escobar". "La mayor parte de la industria latinoamericana está basada en la transferencia cle técnicas de un país más desarrollado, sin que vaya acompañada de mayor investigación, ni espíritu científico en esa adaptación; ello provoca en pocos años, no solamente una disminución de la productividad relativa de la industria en sí, sino que, sus productos son eliminados

\footnotetext{
20alboa, Manuel, Discurso en la Conjerencia sobre la aplicación de la ciencia y la tecnología de desarrollo de América latina, unesco, 1965.

23Escobar, I., El científico en el desarrallo de América latina, Conferencia sobre la aplicación de la ciencia y la tecnología al desarrollo de América latina, unesoo, 1965.
} 
del mercado por la aparición de otros nuevos de mejor calidad, a menor costo. Ello lleva al Estado a establecer sistemas impositivos y aduaneros que permitan a la industria del país en desarrollo su sobrevivencia y, en esas condiciones, la producción, en lugar de incrementar las rentas del pais, contribuye a su estancamiento. Al mismo tiempó el técnico que, en los tiempos en que se instaló la industria, se hallaba al día en su especialización, pierde su espíritu creativo, transformando su labor en rutina o emigrando, si tiene mayores aspiraciones". Este proceso ha sido estudiado recientemente por Martin ${ }^{24}$ para la Argentina, uno de los países más industrializados de la región. Refiriéndose al proceso de industrialización por sustitución de importaciones mediante el trasplante de tecnologías' dice: "Los limites de este modelo se adivinan: la ausencia de un esfuerzo de investigación y de clesarrollo técnico impide ese encadenamiento de innovaciones que elevan la eficacia de los procesos productivos $y$, más todavía, modelan al hombre de la industria moderna".

Lo que acabamos de ver no significa, por supuesto, que no sea posible usar el conocimiento tecnológico y científico disponible en los países desarrollados. Todos los países del mundo, y en particular los más adelantados, utilizan para su progreso los resultados de la actividad científica que se realiza fuera de sus fronteras nacionales. Lo que sí significa, es que el traspaso eficiente de tecnologías sólo se puede efectuar si el país recipiento ha alcanzado también un alto grado de desarrollo científico. Las razones son obvias. A.nadie se le ocurre pensar que para elevar el nivel cultural de una región de analfabetos es suficiente instalar una biblioteca provista de los mejores clásicos de la literatura mundial; de la misma manera, es absurdo imaginar que un pais atrasado científicamente pueda usar y adaptar a sus necesidades especificas los resultados de la actividad intelectual más complejả y sofisticada que ha producido la humanidad, como es la ciencia moderna.

El error proviene principalmente de ignorar el carácter esencialmente dinámico de la actividad científica y tecnológica. No existe un cuerpo estable de conocimientos del que se puede disponer en cualquier momento, en forma de recetas técnicas para resolver los problemas materiales que enfrentan los paises subdesarrollados. La investigación científica $y$ tecnológica produce una enorme masa de material continuamente cambiante, que abre cada día nuevos campos al conocimiento y nuevas posibilidades, y que convierte rápida-

3'Martin, J. M., Blocage de developpement el industrialization par substitutions d'importations - L'exemple de l'Argentine. Revve Tiers-Monde, T. vut, No 30, 1967, pp. 503-515. 
mente en anticuados los procedimientos técnicos más avanzados. La selección de los procesos de producción más adecuados a las condiciones particulares de cada país sólo puede hacerse sobre la base, no solamente de un conocimiento exhaustivo de las condiciones locales sino también, y fundamentalmente, de una comprensión clara de los resultados, las tendencias y los posibles desarrollos futuros de la investigación científica y tecnológica. Refiriéndose a cste tema, Powell ${ }^{25}$, premio Nobel en física en 1950, dice: "Aunque puede ser verdad que muchos de los problemas más graves (de los países subdesarrollados) se pueden resolver mediante la aplicación a nuevas situaciones de principios conocidos, es necesario destacar que tales aplicaciones requieren una imaginación científica creativa de primer orden". A. King $^{26}$, uno de los científicos más clestacados de Gran Bretaña, y que ha influido profundamente en la política científica de clicho país, dice también: "Aun una nación pequeña requiere en su interés nacional, que el apoyo a la investigación fundamental sea suficientemente amplio como para proporcionar al pais una comprensión clara del significado de los nuevos avances científicos. Sin esta importante, aunque no siempre obvia exploración, se perderán las oportunidades de progreso tecnológico y faltarán los científicos con la preparación necesaria para futuros desarrollos. La falta de investigación fundamental suficientemente amplia en un pais, es un medio seguro de lograr que sus procesos industriales se vuelvan eventualmente obsoletos". La conclusión evidente es que solamente investigadores científicos y tecnológicos en actividad, pueden hacer que el proceso de transferencia y adiatación de tecnologías desarrolladas en los países adelantados no se convierta en un factor más de estancamiento económico y social.

En lo que precede me he refericlo a la necesidad del progreso científico de América latina, para poder adaptar a sus necesidades específicas los resultados de la ciencia y la tecnología de los países más avanzados. Esto implica, naturalmente, el supuesto de que en los países desarrollados se crean procesos tecnológicos que, aunque sea necesario adaptarlos a las condiciones locales, se pueden aplicar.a satisfacer las necesidades de la región. Esto, sin embargo, no es más que una parte del problema. Existen campos fundamentales de la tecnologia en los cuales la investigación que se realiza en los países industrializados no solamente no es útil a los países subdesarrollados, sino que incluso resulta perjudicial para sus intereses econó-

xpowell, C. F., The Science of Science, Edited by M. Goldsmith and A. Mackay, Penguin Books, 1964, p. 98.

×King, A. op. cil., p. 145. 
micos, por lo menos a corto y mediano plazo. Uno de estos campos, que es vital para los países de América latina productores de materias primas, es el de los recursos naturales.

En los países altamente industrializados gran parte de la investigación tecnológica relacionada con las materias primas está dirigida a reemplazar los materiales naturales por otros sintéticos, disminuyendo así su dependencia de las fuentes de producción de aquéllos. Algunos casos, que por otra parte tuvieron consecuencias serias para las economías de ciertos países de la región, son bien conocidos. Hasta la Primera Guerra Mundial alrededor de dos tercios del ingreso nacional de Chile provenía de la explotación de sús depósitos de salitrè. Al finalizar la guerra, el perfeccionamiento del proceso HaberBosch para producir nitratos fijando el nitrógeno del áire, desplazó el salitre de los mercados mundiales, provocando un gravísimo deterioro de la economía chilena. Las investigaciones para reemplazar el caucho natural por un sucedáneo, comenzaron en Europa a principios de siglo, y fueron impulsadas por las necesidades generadas durante las dos guerras mundiales. La producción comercial en escala importante comienza al finalizar la Segunda Guerra Mundial, y en 1964 el calucho sintético representó el 59,7 por ciento de la producción total. Los abrasivos naturales han sido reemplazados casi totalmente por productos sintéticos; los materiales plásticos están reemplazando no solamente lit maclcra, los productos cerámicos, etc., sino también varios metales en algunos de sus usos, y las fibras sintéticas han reemplazado en gran medida a las naturales en la industria textil. Se podría seguir con una lista realmente interminable de ejemplos, pero es suficiente decir que la ciencia moderna está en condiciones de producir sucecláneos de casi cualquier producto natural, siempre que disponga de la energía suficiente.

Uno de los resultados de esta política tecnológica de sustitución, menos evidente pero igualmente peligrosa para los países subdesarrollados, es el efecto que sobre el precio de las materias primas ejerce la simple amenaza ò posibilidad de sustitución. En efecto, como señala Aymans, ${ }^{27}$, “. . las innovaciones tecnológicas, particularmente en las técnicas de ahorro y de sustitución de materiales, son provocadas sobre todo por la tendencia.al alza de los precios de las materias primas pertinentes. A tal punto que hay casos en los que los propios productores de artículos primarios han hecho todo lo posible para que no suban los precios de sus productos... Ocurre muchas veces que

${ }^{27}$ Aymans, G. H. P., Tendencias de la tecnología relativas a la utilización de los recursos naturales, UNEsco, es/0765, 131 APs, 16 (ws), p. 29. 
ya se ha encontrado un sucedáneo o se ha inventado una técnica de ahorro de material, pero no se aplican mientras el precio del producto natural no suba tanto como para justificar el empleo del nuevo producto o de la nueva técnica. En otras palabras, cl hecho de que una materia prima escasee o de que su precio suba no significa que se le ofrezcan mejores perspectivas. Al contrario, cuanto más falte y más cara se torne, mayor es el riesgo de que se la reemplace enternmente". La industria del cuero es un ejemplo de esta situación. La firma Dupont produjo recientemente un sucedáneo del cuero que no se fabrica todavía por su alto costo, pero si el precio del cuero sigue subiendo, este material terminará por reemplararlo totalmente.

La única posibilidad que tienen los países de América latina de defenderse de los efectos cle esa política de sustitución y ahoró de materias primas naturales, es clesarollar activamente su propia investigación tecnológica en ese campo. Muchos productos naturales son reemplazados porque Ios sucedáneos tienen características físicas o de composición más homogéneas o porque sus técnicas de elaboración permiten ajustar más rápiclamente el volumen de producción a la demancla. La investigación tecnológica, mediante una mejor tipilicación y clasificación de esos productos y a través del estudio le procesos de producción más flexibles, puede ayudar a reducir, por lo menos en parte, esas desventajas. El desarrollo de nueros usos para los productos naturales es otro de los campos abiertos a los investigatolores de la región.

Una cle las tareas más importantes que clebe cncarar la investigación tecnológica en América latina es la creación de nueros recursos naturales. La expresión parece paradójica, pero responde a la realiclad. Los recursos llamados natumales no son estrictamente tales; son esencialmente el producto de la investigación científica y tecnológica. Como ya hemos visto, los clepósitos de salitre de Chile se convirtieron en recursos naturales cuanclo el progreso de la tecnología agraria llevó a la fabricación de fertilizantes nitratados, y' dejaron casi de serlo cuando el mismo progreso tecnológico permitió la utilización del nitrógeno clel aire con ese fin. Los depósitos de mineral de hierro con alto contenido de sílice (taconitas) cle los Estados Unidos, no eran aprovechables hasta hace dos clécaclas. Cuanclo los yacimientos de alto contenido de hierro comenzaron a agotarse, los científicos norteamericanos desarrollaron procesos tecnológicos que permitieron la utilización de esas taconitas en la industria słderúrgica. Hasta hace pocos años se consideraba que la producción de aluminio sólo podía efectuarse económicamente a partir de bauxitas con alto contenido de alúmina y menos de $6 \%$ de sílice. Algunos cle los países industrializados que no contaban con acceso fácil a los recursos de bauxita 
clel mundo, como Rusia y Alemania, cstudiaron y desarrollaron técnicas que permiten ahora producir aluminio a partir de materias primas naturales que no se consicleraban recursos hasta hace pocos años, neutralizando así el virtual monopolio de las bauxitas de alta ley que ejercen algunas de. las grandes potencias industriales. Los yacimientos de cobre porfírico clel tipo de los que constituyen la mayor fuente de recursos cle Chile, no eran explotables, por su baja ley, hasta hace pocas décatlas. El enorme aumento en la clemanda de cobre que produjo el crecimiento de la inclustria, llevó a que los científicos de las. grandes potencias industriales crearan tecnologias que permiten ahora cxplotar económicamente esos yacimientos. Los casos citados son solamente algunos muy conocidos dentro del campo de los recursos no renovables, pero la lista pollía alargarse considerablemente con ejemplos de utros sectores dic la producción, como la agricultura. Además, el crecimiento y la diversificación casi explosivas de la industria modema generan cada día la necesiclad de utilizar nuevos materiales. Sustincias que hasta hace pocos años eran poco mís que curiosidades cle liboratorio, como el uranio, el selenio, el germanio, el torio,- etc., se han transformado en pocos años en integrantes de la, compleja maquinarial de producción, requiriendo la búsqueda de nuevos recursos naturales que los contengan.

En conclusión, así como lat investigación científical y tecnológica "neutralizal", para usar la expresión cle Zimmermanes, recursos natumales al sustituirlos, también puecle crearlos. Es sorprendente observar, sin embargo, que prácticamente en todos los casos la investigación tencliente al aprovechamiento de nuevos recursos naturales ha sido efectuaclia en los países industrializados, respondiendo a sus propias necesidacles y conveniencias. Los países productores de materia prima, como los de América Intina, se han limitado simplemente a explotar aquellos recursos que clemanclan los países desarrollados en función de su experiencia tecnológica con determinado tipo o forma de prèsentación del mismo. Esta falta casi absoluta de creatividad en el campo más vital para la economía de los países cle América latina, es una demostración palpable clel colonialismo económico e intelectual a que estos países están sometidos. El cleterioro continuo de los términos del comercio exterior cle los países cle la región, es no solamente la consecuencia natural del progreso tecnológico, que tiende a disminuir el valor de la materia prima en los procesos de producción, sino también, y en gran medida, el resultado de la carencia de

Zimmerman, E. W., World resources and industries, New York, 1951. 
una capacidad científica que les permita afrontar en forma creativa $y$ dinámica ese problema.

Veamos ahora la segunda proposición, la que dice que los países subdesarrollados necesitan técnologías de producción "intermedias", con mayor uso de mano de obra, y por lo tanto pueden resolver sus problemas con una ciencia también "intermedia", de menor nivel que la de los países adelantados. Creo que los argumentos que acabo de dar, en relación con la posibilidad de obtener el desarrollo científico y tecnológico en base al trasplante y a la copia de lo que se hace en los países adelantados, son también suficientes para móstrar el carácter poco realista de esa proposición. No obstante, y teniendo en cuenta que se trata de una hipótesis bastante favorecida en algunos círculos políticos y económicos de la región, conviene analizarla con más detenimiento. El primer lugar, se comete el error de creer que un proceso de producción que implique menor uso relativo de capital tiene necesariamente un menor nivel tecnológico. Esto no es siempre cierto, como lo indica muy claramente Aymans ${ }^{20}$ : ". . las llamadas técnicas avanzadas se clenominan así simplemente porque la práctica general parece indicar que se califique asi a todo sistema de producción que se traduzca en un aumento del reridimiento (o del valor añadido) por cabeza, del factor trabajo asociado al mismo. Habitualmente el elevado rendimiento por cabeza es una consecuencia de la mayor canticlad de capital empleado por trabajador y no de la superioridad tecnológica o económica respecto a otros medios". El error proviene en gran parte de suponer que el aprovechamiento de tecnologías de mayor incidencia de mano de obra se logra usando procedimientos y equipos de producción anticuados, descartados ya por los países más desarrollados. La solución, sin embargo, no puede ser ésa, salvo en casos muy especiales. Para que los procesos de producción "intermedios" contribuyan realmente al progreso de la región, y puedan ser competitivos con respecto a los que se utilizan en otros países, deberán incorporar todos los adelantos de la tecnología moderna compatibles con el tipo de proceso elegido. Esto requiere una alta capacidad de investigación científica y tecnológica en la región, porque son problemas que no interesan, y por lo tanto no se estudian, en los países altamente industrializados. Es necesario distinguir, además, entre el mayor o menor grado de complejidad y sofisticación de los equipos que se pueden usar en los procesos de producción, y el nivel de capacitación técnica que se requiere para decidir entre las muchas

Pymans, G. H. P., Tendencias de.la tecnologia relativas a la utilización de los recursos naturales, uNes $\infty$, es/0765. 131 APs. 16 (ws), p. 17. 
alternativas posibles. La selección de los procesos de producción más convenientes para los distintos países de América latina debe considerar un número tan grande de variables, que solamente la pueden hacer científicos'y tecnólogos del más alto nivel, que conozcan además profundamente las condiciones particulares de la región.

Por otra parte, es fundamental tener en cuenta que la necesidad de usar en algunos campos de la producción las llamadas tecriologias "intermedias", no es más que una parte, y no la más ìmportante o la más difícil, de los múltipes problema científicos $\mathrm{y}$ tecnológicos en los que América latina deberá buscar sus propias soluciones. Para clemostrarlo basta mencionar unos pocos. Gran parte de los habitantes de Amériça latina viven en la zona tropical, y otra porción considerable está establecida en zonas cuya altitud media sobrepasa los 3.000 metros sobre el nivel clel mar. Los problemas específicos de todo tipo que crean esas condiciones ambientales -sanitarios, de producción agrícola e industrial, de comunicaciones, etc.-, no han sido hasta alhora investigados en cletalle simplemente porque los países desarrollaclos están en zonas clel munclo que no los presentan. Sin embargo, el pleno dominio del medio ambiente físico es una condición "sine qua non" del progreso de una sociedad.

La discusión precedente se podrá resumir dicienclo que la problemática clel subdesarrollo plantea uno de los desafíos intelectuales más grandes que una sociedad haya enfrentaclo en la historia. Como en toclos los grandes desafíos históricos anteriores, las soluciones las pueden dar solamente los protagonistas; $y$ esto es tan cierto en el terreno de la creación cientílica como en todos los otros campos de la actividiad humana.

Admitida la necesidad de que los países de América latina desarrolleń una ciencia y una tecnología propias de alto nivel, se plantea el difícil problema cle saber cómo puede generarse deliberadamente en una socicdad atrasada, el potencial de creación cientifica y tecnológica que poseen las sociedades más desarrolladas. No se trata de lograr simplemente que exista actividad cientifica. Esta existe $y$ ha existido siempre en toda sociedad civilizada, porque como el arte, es uno de los productos primarios de la actividad humana. Se trata de entrar en lo que se ha dado en llamar la Revolución Científica y Tecnológica, es decir, en ese proceso autocatalíticos en el cual el progreso acelerado de la ciencia se traduce espontánea y automáticamente en un mayor bienestar de la sociedad, el que a su vez repercute sobre la 
actividad cientifica estimulándola. Este es el proceso que hoy permite a los países adelantados incrementar su bienestar a un ritmo nunca igualado en la historia.

Es evidente que para saber qué concliciones se requieren para que una sociedad se incorpore a la Revolución Científica, en el sentido amplio en que la hemos definido, es necesario saber pirimero qué condiciones se dieron para que otras sociedacles lo hayan hecho en el pasado, especialmente en cl pasado reciente. No se trata ahora de intentar un análisis cletallaclo, que por otra parte sólo podrían hacerlo historiadores y sociólogos que aclemás tuvieran un conocimíento acabado de la historia de la ciencia, sino solamente de ver, muy esquemáticamente, cuáles fueron las condiciones generales que permiticron o impulsaron el proceso.

Los historjadores modemos consideran que la Rerolución Cientifica se genera y adquiere su carácter delinitivo on el periodo comprendiclo entre los años 1500 y 1700:31. En este períndo fuc fundamentalmente una revolución intelectual que cnseñó a los hombres a ver el mundo en forma diferente, a pasar "de un munclo de cosas orclenadas de acuerclo con su maturale\% icleal, a un mundo de eventos que se desarrollan en un constante mecanismo de antes y despućs"'a. Solamente clespués, a fines del siglo dieciocho, con la Revolución Industrial, esta nueva forma cle pensar se incorporal al quehareer praíctico de la socieclacl, condicionanclo toclo su desarrollo futuro.

La Revolución Inclustrial es uno de los procesos más estuliados de la historia, y aunque se conocen bastante bien las calusas generales que la produjeron, no existe todaríit un acuerelo completo sobre la importancia relativa de los clistintos factores que intervinieron en ella. Para nuestros fines, sin embargo, es suficiente señalar algunos puntos esenciales. En primer lugar, la Revolución Inclustrial comentó en Inglaterra, y sólo posteriormente se extendió a otros países de Furopa: La importancia de este hecho radica en que, como señala Eric Hobsbawm"2, "cualquiera que sea la causa cle este avance de Inglaterra, no fue ciertamente su superioridacl cientifica y tecnológica". En efecto, las ciencias naturales, la física y las matemáticas estaban mucho más adelantadas en Francia que en Gran Bretaña, y los sistemas educacionales de Francia y Alemania habian alcanzado niveles muy superiores a los de ese país ${ }^{33}$. Las necesidades tecnológicas de los comienzos de la p. 133 .

${ }^{30}$ Bronowsky, J., Ana Mazlish B., The Western intellectual iradition, Pelican Books, 1963,

31Bronowsky, J. The common sense of science, London, 1951.

32Hobsbawm, E. J., The agc of revolution: 1789-1848, New York, 1964, 1). 47.

${ }^{23}$ Op. Cil., p. 47. 
Revolución Industrial en Inglaterra fueron relativamente modestas, y pudieron satisfacerse con la capaciclacl inventiva de artesanos inteligentes. Es muy significativo el hecho de que la máquina rotativa de vapor, el elemento tecnológico más complicado que se usó en ese período, fue inventada por Watt en 1784 , más de 30 años antes de que Carnot desarrollara en Francia los principios teóricos que constituyen la base del funcionamiento de las máquinas de vapor.

Entre los factores más importantes que hicieron posible el comienzo de la Revolución Inclustrial en Inglaterra, se destaca la transformación rcvolucionaria de la agricultura que tuvo lugar desde mediados del siglo dicciocho y que estaba prácticamente terminada al finalizar esc períoclo. Citanclo nuevamente a Hobsbawm ${ }^{34}$ : "La agricultura estaba ya preparada para llevar a cabo sus tres funciones fundamentales en una cra cle inclustrialización: aumentar la producción $y$ lia productividad, de manera cle pocler alimentar a la creciente población no cleclicala a la agricultura; proveer un excédente de mano de obra para incorporarse a las ciudades y a la inclustria; y proporcionar un mccanismo pilla la acumulación clel capital a ser usaclo en los sectores mís modernos de la economia". El otro factor decisivo fue li creación, it través cle lit expansión creciente de la actividad mercantil, de una clase gobcrnante enriquecida que se interesaba por promover el desarrollo cconómico. "El' éxito logrado por Inglaterra en cliseñar $y$ aplicar las nuevas fueras mecánicas fue a la vez causa y consecuencia de lit visión comercial amplísima del conjunto de la. clase media y' de gran parte de la clase alta" 35 .

Surge claro, entonces, que si bien la Revolución Científica que comienza en el siglo dieciséis fue esencial para posibilitar la Revolución Inclustrial, ésta no se hubiera probablemente producido de no existir otros factores socioeconómicos que hicieran posible la utilización de los productos de la creación científica a los fines del progreso de la. socieclacl. Vale la pena recordar que la ciencia griega de la antigüeclacl, particularmente en el período alejandrino, con hombres como Euclides y Arquímedes, había llegado a un nivel intelectual que no cstaba lejos del alcanzado en los comienzos de la Revolución Cientifica moderna. Las causas de su paralización y decadencia sin efectos visibles en la sociedad de su tiempo, deben probablemente buscarse en la estructura misma de esa sociedad que, basicla en la esclavitud, no tenía estímulo suficiente para buscar su progreso material en el desarrollo de la tecnología.

sOp. Cill., p. 45.

${ }^{35}$ Colc, G. D. H., Introducción a la historia económica, Fondo de Cultura Económica, México, 1957, p. 60. 
Lá Revolución Industrial comenzada en Inglaterra se propaga a parte de Europa y a los Estados Unidos, y para la segunda mitad del siglo diecinueve ya ha producido la división del mundo en dos bloques: el integrado por los países cuyo desarrollo crece acelerada. mente utilizando todos los recursos de la ciencia y de la tecnología, y el formado por el resto de la humanidad, que permanece en la pobreza y el atraso. Durante el período que sigue y que llega hasta nuestros días, sólo un pequeño grupo de países de los que no se beneficiaron de lo que podríamos llamar la primera Revolución Industrial, logró dar el salto cuantitativo y cualitativo que les permitió iniciar el proceso de desarrollo acclerado utilizando los recursos de la ciencia en todos los campos de la activiclad social. Esos países son Japón, Rusia y más recientemente China. En estos casos la liistoria es bien conocida. Japón, ante el desafío de las potencias occicleritales materializado por la expedición del Comodoro Perry en 1853, responde modificando toda su estructura politica y social para poder modernizarse incorporando la capacidad creadora cle la ciencia y la tecnología occidentales. En 1869 queda aboliclia definitivamente la estructura feudal del país; en 1871 se inaugura un sistemal educicional moderno controlado por el Estado, y al finalizar el siglo, Japón emerge como una de las grandes potencias mundiales. Los casos de Rusia y China no necesitan mayor comentario: la incorporación de la ciencia y lạ tecnología como motores de adelanto social se produce como consecuencia de revoluciones que modificaron profundamente la estructura social y política cle esos paises.

Las conclusiones de este brevísimo análisis se pueden resumir fácilmente. El proceso que hemos denominado Revolución Científica y Tecnológica se ha producido siempre como respuesta a necesidades fundamentales explicitadas por la sociedad. No basta que existan necesidades; es necesario que la sociedad se haga consciente de ellas y se proponga deliberadamente satisfacerlas. El desafío de occidente fue sufrido por todos los países de Asia, pero solamente Japón reaccionó enfrentándolo con éxito. La necesidad de terminar con el atraso, el hambre y la miseria en gran parte del mundo subdesarrollado no basta para generar progreso, mientras esas sociedades no se propongan, consciente y deliberadamente, satisfacerla.

El progreso científico y tecnológico es entonces un elemento esencial del desarrollo, pero no puede producirse aislado, independientemente de los factores sociales y políticos que condicionan una comunidad. Esto surge, por otra parte, de la naturaleza misma de la ciencia moderna. En el pasado, al comienzo de la Revolución Científica, la investigación podía ser realizada por hombres relativamente aislados, 
con pocos medios materiales, y por lo tanto, era en cierta medida independiente, por to menos en el sentido material, del medio que la rodeaba. En la actualidad la situación es totalmente distinta. La investigación científica y tecnológica requiere medios de una complejiclad y magnitud que sólo el conjunto de la sociedad, a través del Estado y de los grandes medios de producción, puede proporcionar. Además, la formación de personal científico en la cantidad y calidad adecuada requiere planes de educación y entrenamiento que aprovechen al máximo los recursos intelectuales de la nación. Una sociedad realiza un esfuerzo de esta magnitud, solamente en la medida en que es plenamente consciente de los beneficios que le reporta.

Tenienclo en cuenta lo que acabamos cle ver, es legítimo preguntarse si, claclo el eştancamiento socioeconómico actual de América latina, se puede hacer algo ahora para impulsar su desarrollo científico y tecnológico. Yo creo que sí. Las fuerzas de cambio de una sociedad no sc generan nunca simultáneamente en todos sus sectores, y el aclelanto relativo de uno de ellos puede ayudar a estimular el cle los otros. Yor otra parte, la concepción y la puesta en marcha de una política de desarrollo científico y tecnológico es una tarea larga y difícil que no puede realizarse de un día para otro. Todo lo que se adelante ahora, aunque sea en el aspecto de su formulación, será tiempo ganatlo cuando se den en la región las condiciones que le permitan entrar clecidiclamente en el camino del progreso.

Entre los objetivos de este breve trabajo no figura, por supuesto, cl de pretender formular una política de desarrollo científico para América latina. En consecuencia, sólo me limitaré a exponer algunas icleas bạ́sicas sobre ese tema.

Una de las primeras preguntas que se formulan los economistas y políticos cle América latina en relación con el problema del desarrolo científico, es el de su posibilidad misma en la región, por·lo menos en el nivel de los países industrializados, teniendo en cuenta su alto costo en recursos materiales y humanos. En apoyo de esta duda se citan las sumas verdaderamente astronómicas que las grandes potencias industriales, como los Estados Unidos y la Unión Soviética, invierten en ese campo. Para plantearse el problema en forma más realista conviene recordar que gran parte de la inversión que realizan esos países se destina a temas de investigación que no ofrecen interés inmediato para los países subdesarrollados, como la carrera espacial, el perfeccionamiento de las armas atómicas y convencionales, etc. Aun 
un país como Francia, de poderío económico mediano, destina gran parte de sus esfuerzos científicos a la creación de una fuarza nuclear.

La clemostración más acabacla de que se puede llegar a un alto nivel científico con recursos relativamente modestos, lo clan Suecia e Israel. Suecia ha clesarrollaclo una ciencia y una tecnología que le permiten competir con los países más adelantados; aun en campos tan "exclusivos" como el de los aviones supersónicos cle guerra. Israel ha conseguido resolver en pocos años la mayor parte de los problemas que le plantea un medio físico.,hostil, mediante la aplicación de su capaciclacl científica. En ambos casos se trata de países cuyo poderío eco. nómico no es mayor que el de algunos países de América latina. El problema, por lo tanto, es más de planificación inteligente que cle meclios.

La planilicación del esfuerzo científico fue uno de los grandes temas cle discusión en los medios académicos antes de la Segunda Guerra Mundial. Como recuerda Price 30 , profesor de Historia de la Ciençia de la Universiclad de Yale: "Para aquellos de nosotros que recordamos los brillantes debates entre los partidarios de la planificación y sus adrersarios, fue bastante sorprendente encontrar que, de cualquier manera, todo estaba planeado al final como resultaclo de la guerra y cle su Gran Ciencia". Esta tendencia a la planificación, que comienza esencialmente como resultaclo de la necesidad de las grandes potencias de declicar todas las energías nacionales al esfuerzo bélico, continuó clespués en los países clesarrollaclos hasta la actualiclad:: "esta época de la revolución técnica se caracteriza por la organización de la "industria de la investigación". En efecto, se constata actualmente que la mayor parte de la investigación que se realiza en el munclo está orientada y canalizada hacia la solución de problemas específicos y predeterminados, dichas soluciones constituyendo los "productos" de esa industria de la investigación" $3 \bar{T}$. Las razones fundamentales de este esfuerzo de planificación son dos: en primer lugar la dificultad de los estados, aun de los más poderosos, de disponer de recursos humanos y financieros para cubrir igualmente todos los campos de la investigación científica, y en segundo término, la creciente comprensión por los gobiernos del papel esencial que la ciéncia y la tecnologia juegan en el progreso social.

En los países cle América latina, la escasez de recursos y la necesidad cle resolver los urgentes problemas que plantea el desarrollo, hacen imperativo la planificación del esfuerzo científico. $\mathrm{King}^{38}$, director cien-

${ }^{a}$ Price, D. J.. The Science of Science, Edited by Goldsmith, M. and MacKay, A., Penguin Books, 1966 , p. 256.

3instilulos latinoamcricanos de investigación tecnológica; tipos, programas y coordinación regional, uNesco, es/0865. 66/Ars - 16 (ws), p. 3.

3*King, A. The Science of Science, Edited by Goldsmith, M. and MacKay, A., Penguin. Broks, 1966, p. 144 . 
tífico de la $\mathrm{OECD}$, dice refiriéndose a ese tema: "Estos países sienten ya la limitación de su disponibilidad de recursos, y sin embargo son plenamente conscientes de las posibilidades que la ciencia ofrece para su futuro... Son estos países los que tienen una necesidad clara e inmediata de una política científica deliberada. Parecen existir dos posibilidades complementarias: concentración del esfuerzo en unas pocas direcciones principales, y extensión de los recursos a través de una participación selectiva en los planes internacionales de investigación". Estos conceptos se refieren a los países de Europa de menor desarrollo científico, relativo, pero son totalmente aplicables a los nuestros.

La planificación del esfuerzo científico sólo puede hacerse, por supuesto, en el marco más amplio dé la planificación del desarrollo. Esta planificación clebe incluir una política definida de orientación de la investigación científica que puede resumirse en tres etapas:

a) Determinar en orden de prioridades los problemas de los países y las necesidades de acuerdo con la estrategia de desarrollo nacional;

b) Formular esas necesidades de orden económico y social en términos técnicos, transformando los problemas en objetivos concretos cle investigación;

c) Implementación de los rẹsultados de esa investigación incorporíndolos al sistema económico activo. La elaboración de un plan de esta naturaleza sólo puecle hacerse con la participación activa de científicos en los organisinos encargados de la planificación del desarrollo.

En los círculos académicos de América latina existe todavía mưcha resistencia a aceptar la planificación de la actividad científica por el Estaclo. Estal actitud está en buena parte justificada por una larga tradiciớn de interferencias políticas negativas en las actividades académicas de la región. Sin embargo, comọ una cierta medida de planificación es inevitable, aunque sólo sea por la necesidad de asignar prioridades en la distribución de los limitados fondos disponibles, es indispensable que los científicos participen activamente en la elaboración de las políticas de desarrollo. Como se señala en un informe del Instituto Venezolano de Investigaciones Científicas: "Si la ciencia ha de ser como es realidad hoy, un instrumento de desarrollo intelectual y de acción material positiva, su matrimonio con la política, en el sentido noble de la palabra, es inevitable"39. Efectivamente, es la única manera de lograr que la planificación del desarrollo cientifico sea hecho por los científicos, y no para los científicos.

æOperación de algunos organismos nacionales de ciencia y tecnología en América latina, UNESCO, CRPAit, 1965, p. 38. 
El tema de la participación de los científicos en la solución de los problemas clel desarrollo está ligado estrechamente, en mi opinión, a uno de los problemas más importantes que afectan a la ciencia de América latina: la emigración de científicos hacia los países más desarrollados. Para solucionarlo se proponen en general dos tipos de medidas: mejorar sus condiciones materiales de vida y clotarlos de mayores facilidades para su trabajo. Es evidente, sin embargo, que estas medidas, a pesar de ser necesarias, no son suficientes. En efecto, los países de América latina no están en condiciones de competir, en lo que se refiere a condiciones materiales, con las que pueden ofrecer las grandes potencias industrializadas. El problema de la emigración de científicos es esencialmente un problema de motivaciones. Un científico de alto nivel se quedará en América latina, a pesar de las ventạjas materinles que se le ofrezcan fuera de la región, si se siente motiṿado en su trabajo por otros objetivos además de los puramente intelectuales. Esta motivación sólo se la puede dar la convicción cle que su tarea representa un aporte positivo a la solución cle los problemas de la comuniclad a que pertenece.

El campo de la cooperación internacional ofrece también amplias posibiliclades para incrementar la capaciclad cicntífica de los países subclesarrollados, pero me voy' a referir muy brevemente a unó solo de ellos: el de la integración latinoamericana. Las dificultades con que tropieza el esfuerzo de integración de América latina, debido principalmente al cliferente grado de clesarrollo de los países que integran la región, son bien conocidas. En el campo científico y tecnológico, sin embargo, esas dificultades a la acción conjunta son mucho menores. Los problemas son muy similares en todos los países $y$, debido a la naturaleza misma del trabajo científico, existe una comunicación constante entre los investigadores. La cooperación cientílica entre los países de la región puede ser de importancia decisiva para la solución de los problemas técnicos clel subclesarrollo, que por su naturaleza específica, no se estudian en los países más aclelantados.

La tarea de integración científica no necesita empezar simultáneamente en todos los campos de trabajo. Puede iniciarse con proyectos muy concretos de cooperación, que servirian eventualmente como "núcleos de integración" activos.

Estos proyectos o núcleos de integración científica podrían ser, para. comenzar, de dos tipos fundamentales:

a) centros de perfeccionamiento científico y tecnológico que deberían ubicarse en los países que hubieran alcanzado un mayor grado de adelanto en las tareas respectivas, pero en los cuales pudieran participar, en igualdad de condiciones, investigadores de toda la región; 
b)' proyectos de investigación relacionados con planes multinacionales de desarrollo. Como ejemplo de estos últimos se pueden mencionar los planes de desarrollo de cuencas fluviales, y el plan de integración clel grupo de países andinos.

En los últimos años los científicos cle todo el mundo, incluidos los latinoamericanos, participaron en las tareas del año geofísico internacional $y^{\prime}$ en otros planes de cooperación destinadas a resolver problemas que interesan a la comunidad científica mundial. Sería muy interesante que los científicos del mundo subdesarrollado plantearan la posibilidacl de realizar el año científico del subclesarrollo, destinado a estudiar los problemas materiales que contribuyen a mantener en la pobreza y en el atraso a casi clos tercios de la humanidad. 